\title{
Self-Organized Growth of Complex Nanotube Patterns on Crystal Surfaces
}

\author{
Ernesto Joselevich $(\bowtie)$ \\ Department of Materials and Interfaces, Weizmann Institute of Science, Rehovot 76100, Israel \\ Received: 19 April 2009 / Revised: 11 June 2009 / Accepted: 11 August 2009 \\ CTsinghua University Press and Springer-Verlag 2009. This article is published with open access at Springerlink.com
}

\begin{abstract}
The organization of carbon nanotubes into well-defined straight or curved geometries and arrays on surfaces is a critical prerequisite for their integration into nanocircuits and a variety of functional nanosystems. We review the recent development of a new approach to carbon nanotube organization based on self-organized growth directed by well-defined crystal surfaces, or "nanotube epitaxy". We identify three different modes of surfacedirected growth, namely by atomic rows, atomic steps, and nanofacets. Particular emphasis is given here to the combinations of such surface-directed growth with external forces - like those exerted by an electric field or gas flow - for the creation of well-defined complex geometries, including crossbar architectures, serpentines, and coils.
\end{abstract}

\section{KEYWORDS}

Nanotubes, self-organization, surface science, nanostructures, nanofabrication, self-assembly

Carbon nanotubes [1] have unique mechanical, electronic, optical, and thermal properties, which make them attractive building blocks for nanotechnology [2]. However, their organization into well-defined straight or curved geometries and arrays on surfaces remains a critical challenge for their integration into functional nanosystems [3]. A common approach is the controlled deposition of preformed nanotubes from liquid suspension by means of tailored surfaces [4], fluidic flows [5], and electric fields [6]. A different approach is the growth of nanotubes under the influence of electric fields [7] or gas flow [8]. A more recent and promising approach is the organized growth of carbon nanotubes directed by well-defined crystal surfaces [9]. In this article, we review this new approach of surface-directed growth or "nanotube epitaxy", with particular emphasis on its combination with external forces for the creation of well-defined complex geometries.

\section{Introduction: from molecular epitaxy to "nanotube epitaxy"}

Epitaxy generally refers to the "growth of a crystal of one material on the crystal base of another material in such a manner that its crystalline orientation is the same as that of the substrate". Epitaxy of inorganic materials is very important in the semiconductor industry, as well as in many other fields of technology. Molecular epitaxy [10], which refers to the formation of organized molecular layers on crystalline surfaces, has a more complex

Address correspondence to ernesto.joselevich@weizmann.ac.il 
nature than the classical commensurate epitaxy of inorganic crystals, and has been recognized to exist in a hierarchy of different modes: commensurate, semicommensurate (or "coincident") and incommensurate (or "orientational") epitaxy, taking place on atomically flat surfaces; ledge-directed epitaxy, taking place on vicinal surfaces, which are terminated with atomic steps; and graphoepitaxy, taking place on nanostructured surfaces. During the last five years, we have developed different nanotube analogues to some of these epitaxial modes [11], later followed by other groups. These orientational heteroepitaxial modes of nanotube growth should not be confused with continued growth of single-walled carbon nanotubes (SWNTs) [12-14], which may be considered as a mode of nanotube homoepitaxy.

Different phenomena which can now be considered as early manifestations of carbon nanotube epitaxy include the aligned electrophoretic deposition of SWNTs on highly-oriented pyrolytic graphite C (0001) [15], the aligned growth of short SWNTs along the low-index directions of Si (100), Si (111) [16], and Au (111) [17] surfaces, the catalystfree formation of SWNTs along or across step edges of $6 \mathrm{H}-\mathrm{SiC}$ (0001) [18], and the aligned dry deposition of SWNTs on GaAs (110) [19, 20]. In all these cases, however, the nanotubes lay in different directions, and were often too short to be considered as organized arrays. The formation of highly aligned, unidirectional, and dense arrays of long SWNTs on surfaces was first observed to form by chemical vapor deposition (CVD) growth on low-quality C-plane sapphire [21]. The cheap C-plane sapphire wafers turned out to be miscut a few degrees off the C-plane, and the SWNT grew along the atomic steps of the vicinal $\alpha-\mathrm{Al}_{2} \mathrm{O}_{3}(0001)$ surfaces (vide infra). This mode of surface-directed growth could thus be related to ledge-directed epitaxy. Later on, SWNTs were also shown to grow along well-defined directions of a variety of other crystal surfaces by different epitaxial modes, reviewed herein.

\section{Different modes of "nanotube epitaxy"}

Following a rational order from smaller to larger aligning features, we identify three different modes of carbon nanotube epitaxy [11]: lattice-directed epitaxy (by atomic rows), ledge-directed epitaxy (by atomic steps), and graphoepitaxy (by nanofacets).

\subsection{Lattice-directed nanotube epitaxy (by atomic rows)}

Following the first reports of atomic step-templated growth of SWNTs on miscut C-plane sapphire [21], two independent groups reported the observation of aligned SWNT growth on singular A-plane and R-plane sapphire, i.e., $\alpha-\mathrm{Al}_{2} \mathrm{O}_{3}(112 \overline{2})$ and $\alpha-\mathrm{Al}_{2} \mathrm{O}_{3}$ (1102), respectively $[22,23]$, where the SWNTs grow along the [2201] and [11̄01] directions, respectively $[24,25]$. The preferential growth of SWNTs along these lattice directions was attributed to higher charge densities along these atomic rows due to electrostatic and van der Waals forces. The latticedirected epitaxial growth of SWNTs on A-plane and R-plane sapphire was recently used for building field-effect transistors, and a nanotube-on-insulator technology of registry-free nanocircuits was proposed [26]. Lattice-directed epitaxial growth of SWNTs will probably take place on other atomically flat singlecrystal substrates. Recently, for instance, SWNTs were observed to grow on $\mathrm{MgO}$ (001) preferentially along the [110] and [110] directions [27].

\subsection{Ledge-directed nanotube epitaxy (by atomic steps)}

We showed that SWNTs produced by CVD on miscut C-plane sapphire tend to grow along the 0.2-nm-high atomic steps of the vicinal $\alpha-\mathrm{Al}_{2} \mathrm{O}_{3}$ (0001) surfaces [21]. This atomic step-templated mode of nanotube growth leads to the formation of dense arrays of highly parallel arrays of SWNTs, as shown in Fig. 1. The miscut is characterized by an inclination angle $\theta$ and an azimuth angle $\phi$, as defined in Fig. 1(b). The degree of alignment correlates mainly with the miscut inclination angle $\theta$, while the straightness of the nanotubes correlates with the azimuth angle $\phi$. When the step direction is along a low-index direction, such as [1120] or [1010], the nanotubes tend to be straight. However, when the steps run along a high-index direction, then the nanotubes tend to be kinked, with straight segments parallel to the nearest low-index directions. Some nanotubes 
present sharp kinks of exactly $30^{\circ}$, which could be attributed to single pentagon-heptagon defects induced by kinked steps. These defects may involve structural changes along the nanotubes, constituting interesting metallic-semiconducting intermolecular junctions and superlattices. Single-nanotube Raman spectra from these samples exhibit a high intensity of D-band peaks, which indicates a significant loss of translational symmetry, consistent with this picture [28]. Polarized Raman measurements confirm the preferred orientation of the SWNTs along low-index directions [29]. A "wake-growth mechanism" has been proposed [21], where the catalyst nanoparticle slides along the step leaving the nanotube behind as a wake. Recent atomic force microscope (AFM) images of SWNTs grown on well-defined vicinal $\alpha-\mathrm{Al}_{2} \mathrm{O}_{3}$
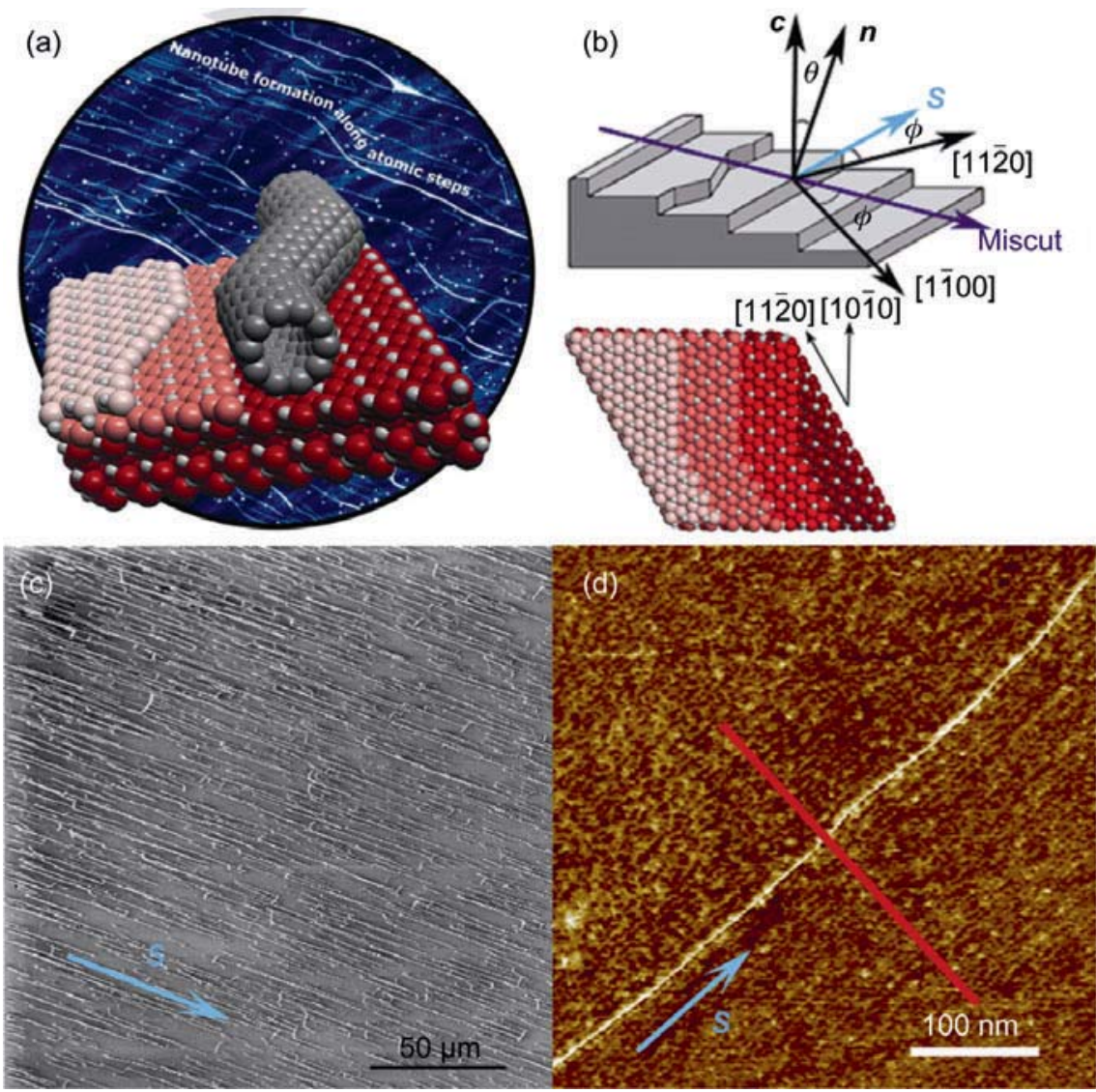

Figure 1 Nanotube formation along the atomic steps of miscut C-plane sapphire, i.e., vicinal $\alpha-\mathrm{Al}_{2} \mathrm{O}_{3}$ (0001) [21]. (a) Model of a kinked SWNT grown along an atomic step on the background of an AFM topographic image of a real sample. (b) Definition of the step vector $\boldsymbol{s}=(\boldsymbol{c} / \mathbf{c}) \times \boldsymbol{n}$, miscut inclination $\theta$, and miscut azimuth $\phi$, where $\boldsymbol{c}$ is the principal lattice vector and $\boldsymbol{n}$ is a unit vector normal to the surface. A top-view model of the vicinal surface is shown below. (c) Large-scale scanning electronic microscope (SEM) micrograph of SWNTs grown on a vicinal $\alpha-\mathrm{Al}_{2} \mathrm{O}_{3}$ (0001) surface. (d) High-resolution AFM topographic image providing a direct observation of the atomic steps on the surface, and an SWNT tightly following one of the steps [29]
(0001) surfaces (Fig. 1(d)), succeeded in resolving the densely packed atomic steps, and confirmed that the SWNTs continuously follow the atomic steps [29].

Ledge-directed nanotube epitaxy was later observed also on miscut quartz [30]. In this case, the surface consists of vicinal $\alpha-\mathrm{SiO}_{2}$ (1101) with steps running along the [2110] direction. SWNTs grown on this surface by CVD form along the steps, similar to those formed on vicinal $\alpha-\mathrm{Al}_{2} \mathrm{O}_{3}$ (0001). The parallel arrays of SWNTs on quartz were used as thin-film transistors. Patterning of the catalyst allowed the formation of spatially selective arrays of SWNTs, and their integration into sub-millimeter thin-film transistors [31, 32]. One important effect of the catalyst patterning is that when the SWNTs grow from the patterned areas onto the clean quartz surface, the nanotube alignment is much better than that without patterning, probably due to the fact that catalyst nanoparticles lying on the path of the nanotube growth can disturb and even terminate the step-directed growth. The formation of aligned SWNTs on vicinal quartz can be catalyzed by nanoparticles of a large variety of metals [33]. More recently, CVD growth on these vicinal quartz substrates from a mixture of ethanol and methanol was shown to selectively produce aligned arrays of semiconducting SWNTs [34].

\subsection{Nanotube graphoepitaxy (by nanofacets)}

"Graphoepitaxy" is a mode of incommensurate epitaxy that generally refers to the orientation of crystals or periodic molecular assemblies by relief features of the substrate, such as steps or grooves, which can be significantly larger than the lattice parameter [35, 36]. Graphoepitaxy of SWNTs was demonstrated on thermally 
annealed miscut C-plane sapphire [11]. When the vicinal $\alpha-\mathrm{Al}_{2} \mathrm{O}_{3}(0001)$ surfaces are annealed at $1100^{\circ}$ $\mathrm{C}$, the thermodynamically unstable atomic steps of $0.2 \mathrm{~nm}$ height bunch together into periodic nanosteps of $1.3 \mathrm{~nm}$ to $4 \mathrm{~nm}$ height. SWNTs grown by CVD on these periodically faceted surfaces generate a variety of morphologies, depending on the shape and surface energy of the nanofacets. As shown in Fig. 2, annealing of a C-plane sapphire miscut in different directions can yield nanofacets with a variety of morphologies (straight or sawtooth) and different surface energies. SWNTs produced on these nanofaceted surfaces (Fig. 3) can have different morphologies (straight, wavy, and ultrastraight). SWNTs grown on metastable nanofacets are especially straight, with angular deviations of less than $\pm 0.5^{\circ}$, for lengths of up to $100 \mu \mathrm{m}$ (Figs. 3(a) and $3(b))$. This can be attributed to the high surface energy of these nanofacets, which makes them particularly sticky toward the nanotubes.

\section{3. "Nanotube epitaxy" combined with external forces}

Combinations of nanotube epitaxy with external forces, such as those exerted by an electric field or a gas flow, offer the possibility of generating more complex carbon nanotube patterns. For instance, crossbar architectures are especially desired for nanoelectronics, as they constitute the basis of most logic and memory elements in computing devices [37]. Although such straight configurations are desirable for the assembly of nanocircuits, one could envisage different devices based on curved and more complex geometries [38], which cannot be obtained by simple mechanisms of aligned growth. Here we will show how "nanotube expitaxy" combined with an external force can yield different types of well-defined complex geometries in a controlled way.

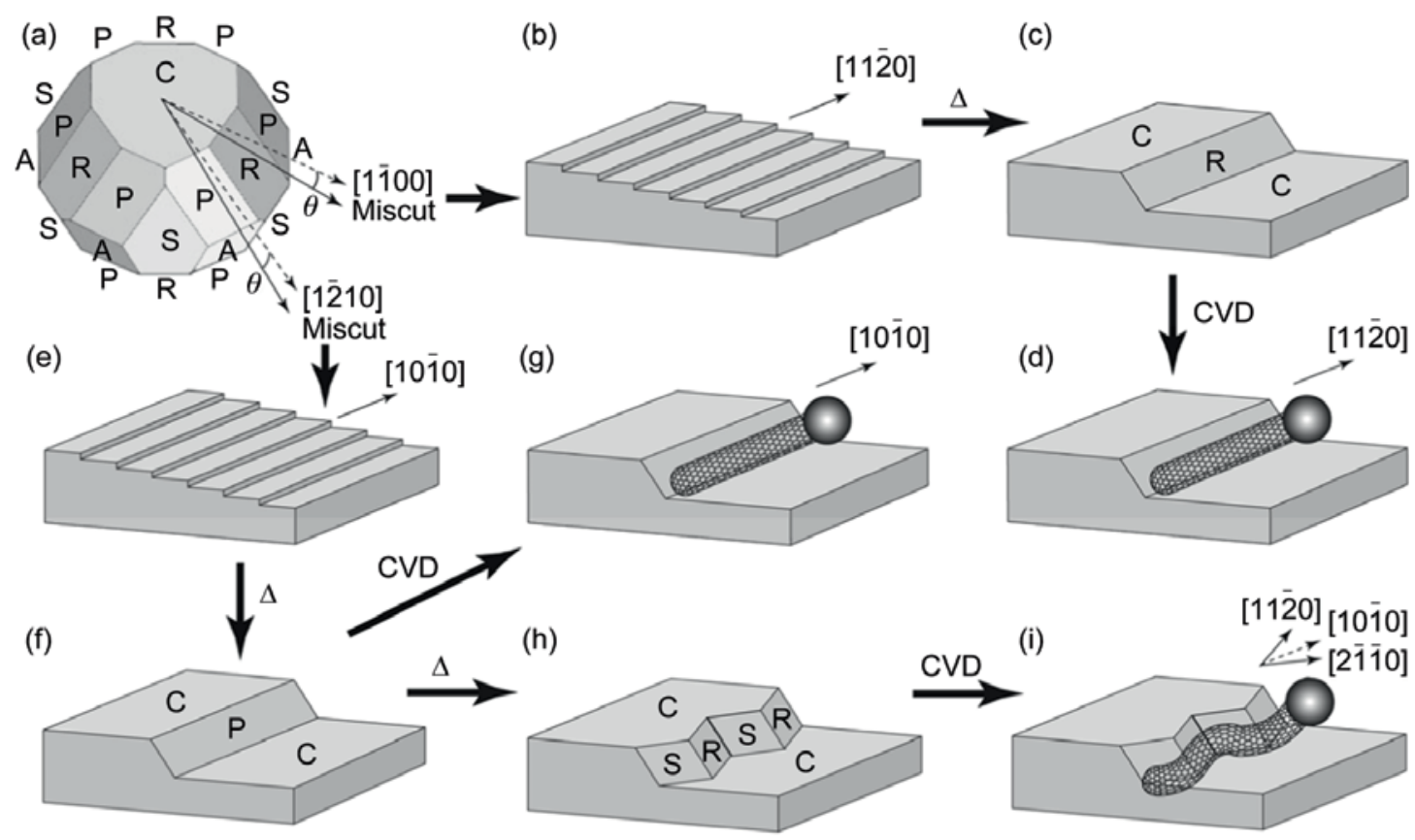

Figure 2 Flow chart describing the formation of possible morphologies of SWNT graphoepitaxy by miscut of C-plane sapphire, annealing, and CVD [11]. (a) Equilibrium shape of $\alpha-\mathrm{Al}_{2} \mathrm{O}_{3}$, with facets $C\{0001\}, R\{1 \overline{1} 02\}, S\{10 \overline{1} 1\}, P\{11 \overline{2} 3\}$, and $A\{1 \overline{2} 0\}$, in order of increasing surface energy. The same drawing is used to show the different miscut directions. (b) Miscut toward [1100] produces a vicinal $\alpha-\mathrm{Al}_{2} \mathrm{O}_{3}$ (0001) surface with atomic steps along [1120]. (c) Annealing leads to R-faceted nanosteps. (d) SWNTS grow straight along [1120] (the ball represents the catalyst nanoparticle). (e) Miscut toward [12̄10] produces a vicinal $\alpha-\mathrm{Al}_{2} \mathrm{O}_{3}(0001)$ with atomic steps along [1010]. (f) Annealing initially leads to metastable P-faceted nanosteps. (g) SWNTs grow straight along [1010]. (h) Further annealing from (f) leads to sawtooth-shaped S/ R-faceted nanosteps. (i) SWNTs grow loosely conformal to the sawtooth-shaped nanosteps, with segments along [11̄20] and $[2 \overline{1} \overline{1} 0]$ 

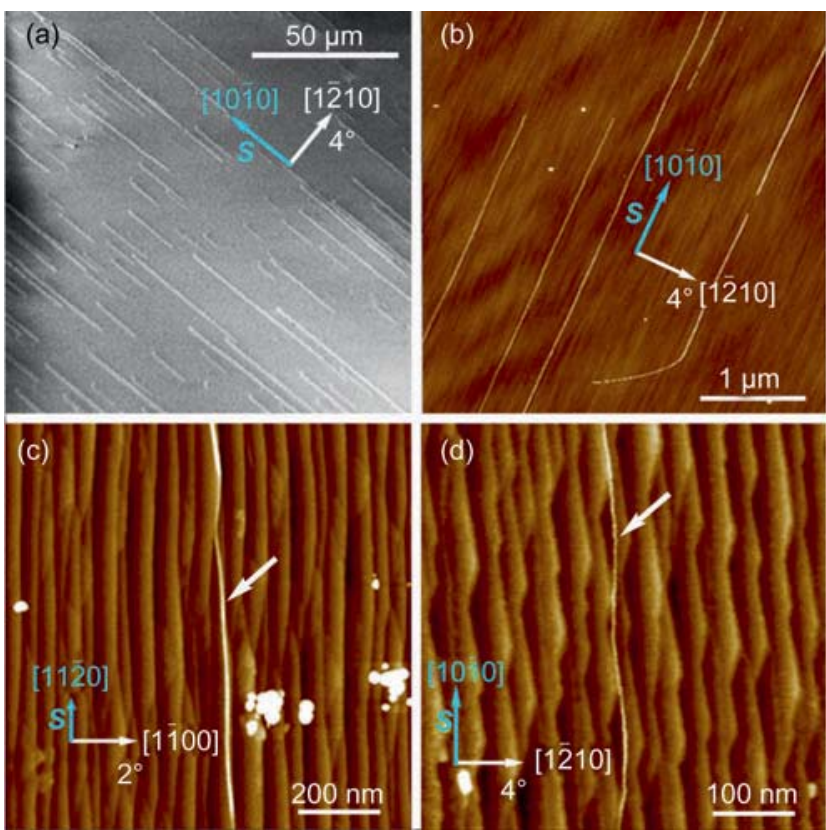

Figure 3 Graphoepitaxial SWNTs on different annealed miscut C-plane sapphire [11]: (a) straight nanosteps along [1010], as in Fig. 2(g), observed by SEM; (b) AFM image of (a), showing the nanosteps; (c) nanosteps along [1120], as in Fig. 2(d); (d) highly faceted sawtooth-shaped nanosteps along [1010], as in Fig. 2(i)

\section{1 "Nanotube epitaxy" combined with an electric field}

Crossbar arrays of SWNTs were spontaneously produced in a single CVD step by simultaneous graphoepitaxy and field-directed growth, perpendicular to each other [39], as shown in Fig. 4. The two alignment mechanisms take place selectively on miscut C-plane sapphire and patterned amorphous $\mathrm{SiO}_{2}$ islands, respectively, without mutual interference, producing dense nanotube grids, with up to 12 junctions per $\mu \mathrm{m}^{2}$. This one-step method of orthogonal self-assembly may open up new possibilities for nanotube circuit integration.

\subsection{Nanotube epitaxy combined with gas flow}

Combined surface- and flow-directed growth has been used to produce crossbar architectures in a similar orthogonal fashion to combined surface- and fielddirected growth [40]. However, the most interesting effect of combined surface- and flow-directed growth is the formation of self-organized nanotube serpentines and coils [41]. Controlled formation of curved nanotube geometries, such as rings and loops, had been previously achieved by directed assembly of pre-formed nanotubes, using affinity templates [42] and microfluidics [43]. On the other hand, surfacedirected growth has so far only yielded nanotubes that are straight and parallel to the atomic steps, atomic rows or nanofacets, although kinked [21] and wavy [11] nanotubes have also been templated by kinked atomic steps and sawtooth nanofacets, respectively, as mentioned above. In all the cases, the alignment was solely determined by the surface, and not affected by external forces such as electric fields or gas flow. These facts were consistent with a surface-bound growth mechanism, where each catalyst nanoparticle slides along the surface guide, leaving the nanotube behind as a wake [11, 21]. Thus, the nanotubes reached the minimum-energy configuration, simply reproducing the shape of the underlying surface features, without a significant addition of complexity to the system.

Carbon nanotube serpentines represent an intriguing new type of nanotube structures which

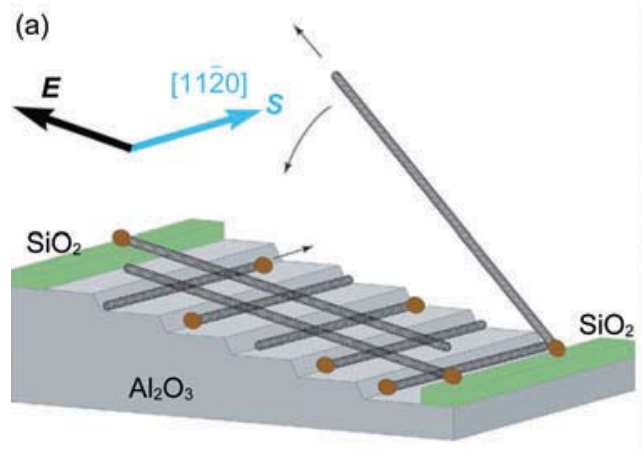

Figure 4 Orthogonal self-assembly of SWNT crossbar architectures by simultaneous graphoepitaxy and field-directed growth, in a single step of CVD [39]. (a) Schematic representation of the process, showing the annealed miscut C-plane sapphire with nanosteps along the [1120] direction, while the electric field is applied perpendicularly to the steps. The nanotubes originating from catalyst nanoparticles lying on the sapphire $\left(\mathrm{Al}_{2} \mathrm{O}_{3}\right)$ grow along the faceted nanosteps, while the nanotubes emerging from the catalyst on patterned amorphous $\mathrm{SiO}_{2}$ stripes grow freely without interacting with the surface, in a way that allows their alignment by the electric field, eventually falling across the nanotubes grown on the sapphire. (b) SEM image of a dense SWNT grid obtained by simultaneous graphoepitaxy and field-directed growth (miscut inclination angle $\theta=4^{\circ}$, field intensity $E=$ $\left.2 \times 10^{6} \mathrm{~V} / \mathrm{m}\right)$

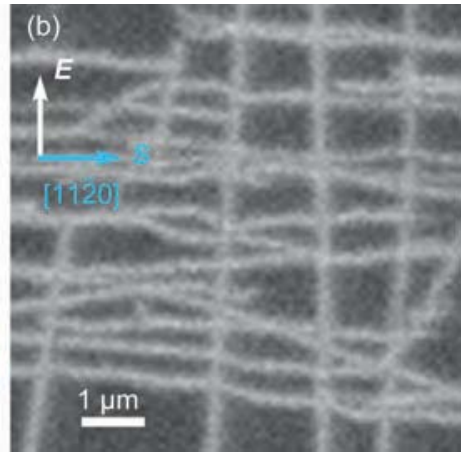

(1) 
are strikingly more complex than those observed previously. We first noticed a few serpentines, such as the one shown in Fig. 5(a), in some of our samples of SWNTs grown on miscut quartz [41]. Each serpentine consisted of a series of straight, parallel, disordered serpentine, which becomes gradually more periodic and regular. Depending on the growth conditions, serpentines can be relatively wide, short, and dense (width $W=10-30 \mu \mathrm{m}$, length $L=20-200$ $\mu \mathrm{m})$, as in Fig. 5(d), or relatively narrow, long, and and regularly-spaced segments, connected by alternating U-turns. The straight segments were parallel to the surface steps of the vicinal $\alpha-\mathrm{SiO}_{2}$ (1101) surface, in the [1120] direction, and the serpentines propagated in the direction of the gas flow, which was perpendicular to the steps. Occasionally, full nanotube loops could also be observed (Fig. 5(a)). The formation of these structures, which were determined by both the surface and the flow, could not be explained by the previous wake-growth mechanism [11, 21]. We hypothesized that the serpentines could result from nanotubes first growing up from the surface, and then adsorbing in an oscillatory fashion along the steps, like a strand of spaghetti falling on a tilted bamboo mat (Fig. 5(g)).

To test this hypothesis and increase the yield of serpentines, we exploited our previous knowledge that an amorphous $\mathrm{SiO}_{2}$ coating inhibits surface-bound growth and promotes the growth of standing nanotubes [21, 39]; we patterned the catalyst nanoparticles (ferritin or evaporated $\mathrm{Fe}$ ) on stripes or islands of amorphous $\mathrm{SiO}_{2}$ parallel to the steps, and then grew SWNTs with the gas flow perpendicular to the steps. This indeed produced a high yield of nanotube serpentines (tens to thousands per sample), as shown in Fig. 5(b). As exemplified in Fig. 5(c), the serpentines usually start from the amorphous $\mathrm{SiO}_{2}$ stripes as a straight segment along the flow direction, followed by a transitional region of
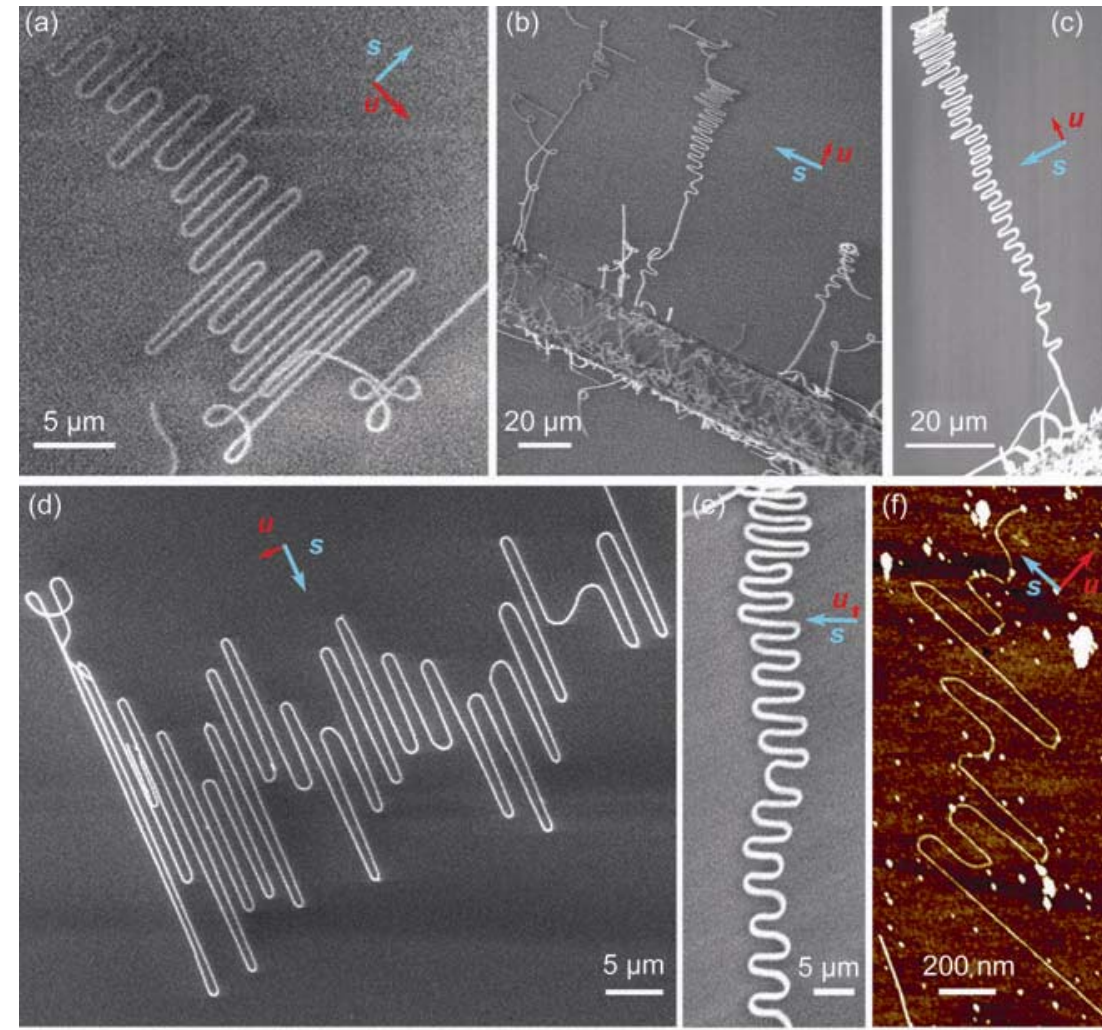

(g)

Amorphous $\mathrm{SiO}_{2}$

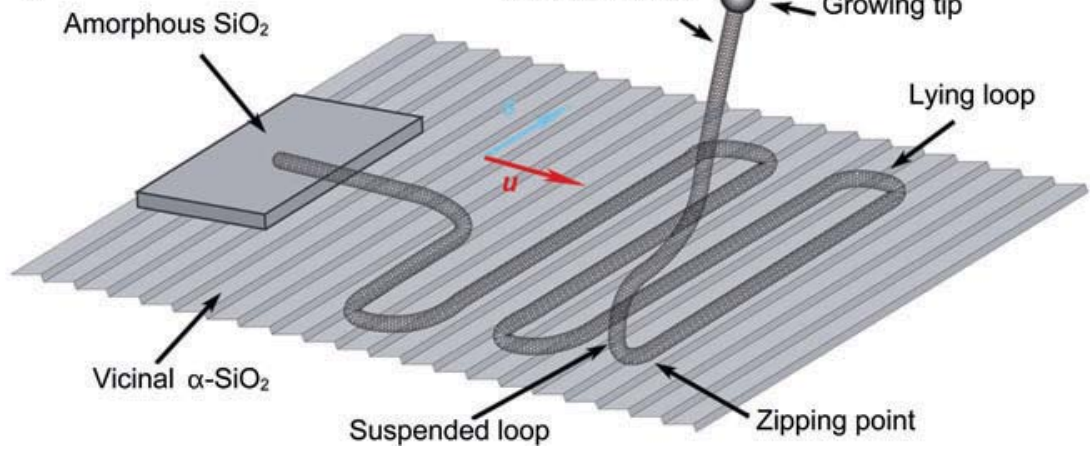

Figure 5 Representative nanotube serpentines obtained under different conditions, observed by SEM (a-e) and AFM (f). The directions of the steps and flow are indicated by the step vector [21] $\mathbf{s}$ and the flow vector $\boldsymbol{u}$, respectively. Unless otherwise stated, standard growth conditions are $\boldsymbol{u}=1000 \mathrm{sccm}, t=10 \mathrm{~min}$, and $\alpha=90^{\circ}$ : (a) occasional serpentine (low yield) grown on bare quartz; (b) several serpentines grown at higher yield from amorphous $\mathrm{SiO}_{2}$ stripes ( $\boldsymbol{u}=500 \mathrm{sccm}, t=30 \mathrm{~min}$ ); (c) a long serpentine $(\boldsymbol{u}=500$ sccm, $t=20 \mathrm{~min}$ ); (d) wide and dense serpentine ( $\boldsymbol{u}=500 \mathrm{sccm}, t=60 \mathrm{~min}$ ), obtained at very high yield from evaporated Fe on amorphous $\mathrm{SiO}_{2}$; (e) long, narrow, and highly periodic serpentine ( $\boldsymbol{u}=250 \mathrm{sccm}, t=20 \mathrm{~min}$ ); ( $f$ ) topographic AFM image of a very small serpentine, average parallel section length $W=280 \mathrm{~nm} \pm 175 \mathrm{~nm}$, average $U$-turn diameter $D=74 \mathrm{~nm} \pm 10 \mathrm{~nm}$, nanotube diameter $d=0.9 \mathrm{~nm} \pm 0.1 \mathrm{~nm}$ (on bare miscut quartz); (g) schematic representation of the "falling spaghetti" mechanism 
highly periodic ( $W=3-10 \mu \mathrm{m}, L=100-1000 \mu \mathrm{m})$, as in Fig. 5(e). The diameter of the U-turns, which is also the spacing between the straight segments, is normally in the range $D=0.5-8 \mu \mathrm{m}$, although a few serpentines can reach very small U-turn diameters $(D$ $=75-100 \mathrm{~nm}$ ), as shown in Fig. 5(f), in which case the U-turns may be kinked at one or two points. As seen in Figs. 5(c)-5(e), serpentines often become slightly wider and denser toward their end.

Considering these observations, we can intuitively describe our proposed "falling spaghetti mechanism" (Fig. $5(\mathrm{~g})$ ) in the following way: a nanotube first grows up from the surface leaning toward the flow direction, and then gradually adsorbs onto the surface by zipping down, from base to tip, preferentially along the surface steps. The zipping point pulls the lower region of the nanotube away from its center of mass, while a drag force pulls its upper region in the opposite sense, causing the formation of a suspended loop tangential to the surface. The zipping propagates farther away along the step until the opposed drag force is enough to derail the nanotube from the step. This causes an instability that leads to a change in the direction of zipping, and the "fall" (adsorption) of the suspended loop on the surface, preferentially in the direction of the flow, thus forming a U-turn. Then, the zipping continues in an antiparallel direction along another step. The same process repeats itself again and again in an oscillatory way, until the entire length of the nanotube has adsorbed onto the surface in the form of a serpentine. Occasionally, if the suspended loop falls against the flow direction, then it makes a round loop, instead of a U-turn, as seen in Fig. 5(a). The whole process is driven by a strong nanotube-surface adhesion, and is countered by the aerodynamic drag and the elastic behaviour of the nanotube.

The gas flow rate and direction have significant effects on the shape of the nanotube serpentines (Fig. 6). As shown in Figs. 6(a)-6(e), increasing the flow rate from 250 to 1000 standard cubic centimeters per minute $(\mathrm{sccm})\left(\mathrm{cm}^{3} / \mathrm{min}\right)$ leads to a gradual increase of the average U-turn diameter (serpentine spacing) from 0.8 to $4 \mu \mathrm{m}$. A higher flow rate $(\sim 1500$ $\mathrm{sccm}$ ) leads to disordered serpentines, and even higher flow rates (2000 sccm and higher) completely inhibit the formation of serpentines, yielding straight nanotubes along the flow direction. Interestingly, suddenly increasing the flow rate from $250 \mathrm{sccm}$ to $1300 \mathrm{sccm}$ after switching off the carbon feedstock gas yields both serpentines and straight flow-directed nanotubes. This indicates that the serpentine "fall" is independent of the nanotube growth, providing further support to our proposed mechanism. The catalysts nanoparticle is usually observed by AFM at the end of the serpentine far from the catalyst patterned islands, providing evidence of a tip growth mechanism. We have not observed a significant effect of using different miscut angles of vicinal quartz. Moreover, we have also observed serpentines on vicinal sapphire, although less frequently than on vicinal quartz.

Changing the direction of the flow with respect to the steps from $90^{\circ}$ to smaller angles has a notable qualitative effect, as shown in Figs. 6(f)-6(i). When the angle between the flow and the steps is between $\alpha=40^{\circ}$ and $70^{\circ}$ (Figs. 6(f)-6(h)), we obtain mostly skewed serpentines with a large number of round loops, and also a significant number of flat coils, each consisting of a series of round loops connected by straight segments (Fig. 6(g)). The skewed serpentines can have left and right U-turns of different diameters (Fig. 6(h)). The diameters of the round loops are relatively uniform for each nanotube coil, varying between 0.5 and $5 \mu \mathrm{m}$ from one nanotube to another. At small angles $\left(\alpha<10^{\circ}\right.$, Fig. 6(i)), the skewed serpentines become mostly straight nanotubes, with occasional sigmoid deformations leaning toward the flow direction.

Besides these flow effects, the U-turn diameter (serpentine spacing) was found to correlate with the nanotube diameter. The nanotube diameter is always constant along each serpentine. Raman studies confirm this finding, and also confirm the fact that each serpentine has both constant diameter and chirality along its entire length, with virtually no structural defects. Detailed statistical studies reveal a roughly linear correlation between serpentine U-turn diameter $D$ and nanotube diameter $d$, with a slightly different factor depending on the flow rate. This correlation in consistent with the higher mechanical stiffness of wider nanotubes, and also with the 
general flow rate effect described in Figs. 6(a)-6(e).

Analysing the shape of the serpentines from a mechanical and aerodynamic point of view allowed us to make some interesting rough estimations regarding the "falling-spaghetti mechanism". First, the diameters of the serpentine U-turns and coil loops (e.g., $D=2.1 \mu \mathrm{m}$ and $3.2 \mu \mathrm{m}$ for the two serpentines in Fig. 3(a)) are more than one order of magnitude smaller than the persistence length of the nanotubes [44] (25 $\mu \mathrm{m}$ and $145 \mu \mathrm{m}$, respectively), and can sometimes be significantly smaller than random loops previously observed [38]. Second, from these U-turn diameters we can estimate the pulling forces attributed to the drag to be $0.17 \mathrm{pN}$ and $0.41 \mathrm{pN}$, respectively. This is three orders of magnitude smaller than the expected driving force for zipping due to surface-nanotube van der Waals interactions [45], i.e., $\sim 2 \mathrm{eV} / \mathrm{nm}=320 \mathrm{pN}$. Third, assuming a classical drag, we can roughly estimate that the zipping velocity for a typical serpentine ranges from 1 to $7 \mathrm{~m} / \mathrm{s}$, which means that the "fall" of the serpentine takes $300-50 \mu \mathrm{s}$, with an oscillation frequency of $50-300 \mathrm{kHz}$. Hence, the nanotube fall is indeed much faster than its growth.

These estimations underscore how far the selforganized serpentines and coils are from equilibrium, and how much energy is dissipated during their formation relative to their final state. This may have insightful implications for the emerging field of non-equilibrium ("dynamic") self-assembly [46, 47], illustrating how dissipative forces and kinetic barriers can lead to the spontaneous generation of much more complex patterns than those generated by the more common equilibrium ("static") self-assembly. Although the serpentines eventually become kinetically trapped, they have the characteristics of dissipative structures [48], because during their formation, their active part is far from equilibrium, in
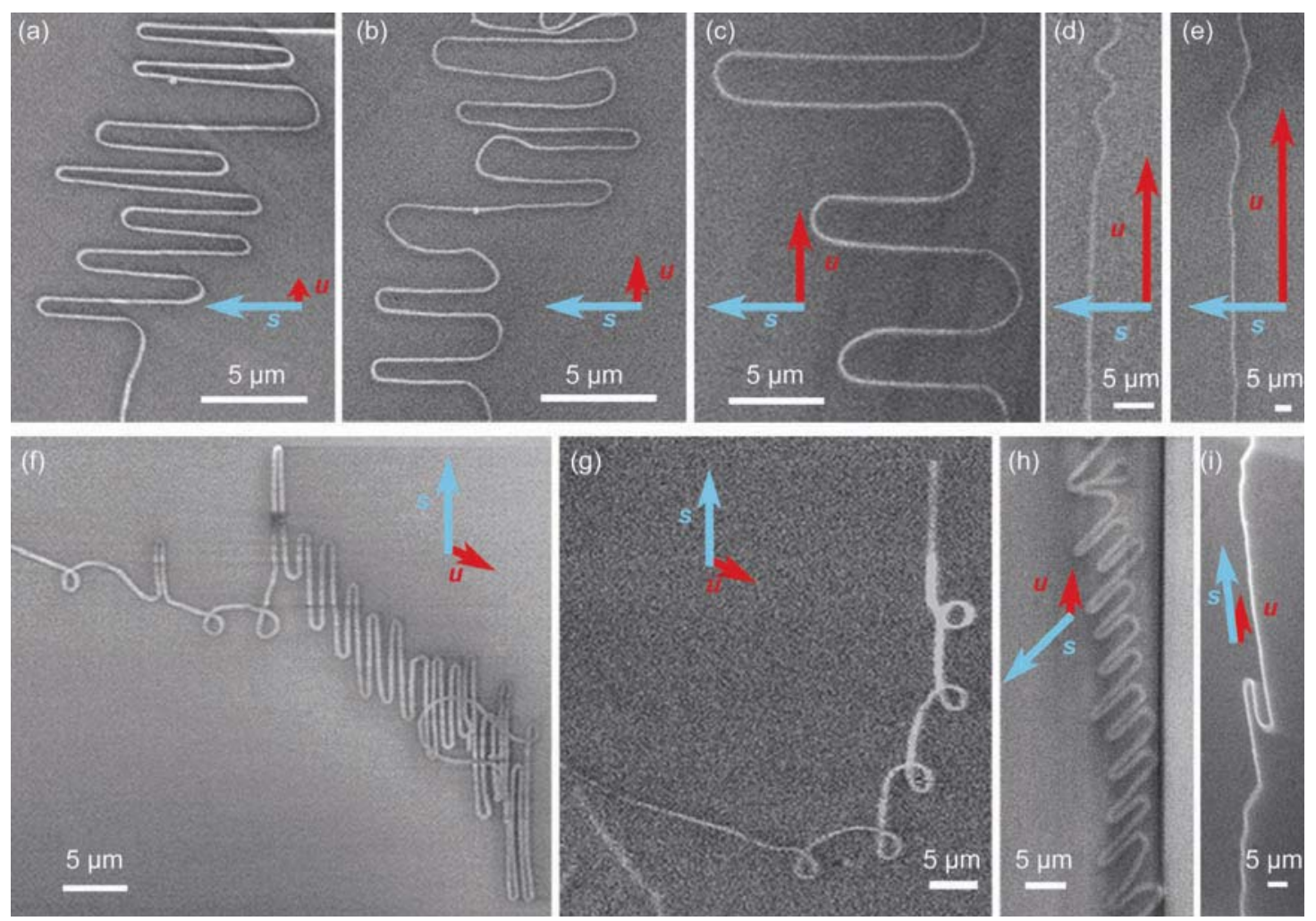

Figure 6 Flow rate and direction effects on serpentine shape. (a)-(e) SEM images of serpentines grown at different flow rates: (a) $\boldsymbol{u}=250 \mathrm{sccm}$; (b) $\boldsymbol{u}=500 \mathrm{sccm}$; (c) $\boldsymbol{u}=1000 \mathrm{sccm}$; (d) $\boldsymbol{u}=1500 \mathrm{sccm}$; (e) $\boldsymbol{u}=2000 \mathrm{sccm}$. As the flow rate increases, the parallel segments become shorter and more spaced (i.e., larger U-turn diameters). Further increasing of the flow rate $(d, e)$ inhibits serpentine formation. (f)-(i) SEM images of serpentines grown with the flow at different angles from the steps: $(\mathrm{f}) \alpha=64^{\circ}$, showing a typical skewed serpentine with several loops $(d=2.0 \mu \mathrm{m} \pm 0.2 \mu \mathrm{m}) ;(\mathrm{g}) \alpha$ $=64^{\circ}$ (same sample as in (f)), showing a nanotube coil of 5 connected full loops; (h) $\alpha=42^{\circ}$, showing a highly skewed serpentine; (i) $\alpha=9^{\circ}$, showing a straight nanotube with sigmoid deformation 
a way that allows for the amplification of fluctuations into coherent oscillations, like in an oscillatory chemical reaction [49]. This system leaves ground for interesting simulations and theoretical studies.

Characterization of the electrical properties of the nanotube serpentines by electrostatic force microscopy (EFM) [50] of entire nanotube serpentines connected at one end revealed that the serpentines are conducting through their full length, with no breaks and no appreciable drop in the potential along the serpentine. The resistance of $10 \mu \mathrm{m}$ serpentine segments was around $2 \mathrm{M} \Omega$, which is still quite high, but this may be due to poor contacts. Resistances across 1-2 $\mu \mathrm{m}$ segments of serpentines can vary from several $\mathrm{M} \Omega$ to as small as $35 \mathrm{k} \Omega$, which is close to the quantum resistance [51]. In principle, entire serpentines could be highly conductive, as recent studies have shown wellaligned SWNTs on similar vicinal quartz substrates to have very high mobilities [52].

From a practical point of view, it is interesting to note that serpentines are a common geometry in many functional systems, such as antennas, radiators, collectors, heating and cooling elements, as well as for lighting, irrigation, etc. Analogously, we can envisage that nanotube serpentines could find a wide range of nanodevice applications, such as in transmitting and receiving antennas of electromagnetic radiation [53] (size-resonant in the terahertz region), heating and cooling elements (e.g., for cooling electronic circuits), and optoelectronic devices (e.g., sensitive IR detectors). In addition, nanotube loops could be the basis for power-generating single-molecule dynamos.

From a more fundamental and general point of view, the spontaneous self-organization of complex structures from simple components is perhaps one of the most intriguing phenomena in vastly different fields, ranging from cosmology [54], geology [55], botany [56], and embryology [57], to chemistry and materials science [46]. It has long been recognized that whereas self-organization near thermodynamic equilibrium tends to attenuate fluctuations, leading to relatively simple geometries, self-organization far from equilibrium can amplify fluctuations into coherent oscillations, leading to much more complex geometries [48]. Notwithstanding its great potential implications for nanotechnology, this universal principle of "order through fluctuations" [48] has not yet been widely applied to the self-assembly of complex structures at the nanoscale [46]. The mechanism of self-organization of carbon nanotube serpentines by combined "nanotube epitaxy" and gas flow constitutes a dramatic demonstration of the principle of "order through fluctuations" at the nanoscale, which opens a new avenue for the controlled formation of nanostructures with welldefined complex geometries.

\section{Conclusions}

Judging by these examples, surface-directed growth of carbon nanotubes or "nanotube epitaxy" seems to be rapidly evolving into an efficient approach for their organization into aligned arrays and complex geometries, including highly straight, kinked, wavy, crossed, serpentine, and coiled. Some of these arrays have been already integrated into thin-film transistors. An intriguing question that remains open is whether all of these modes of "nanotube epitaxy" are indeed incommensurate, or whether there may be at least some degree of commensurism or registry between the surface lattice or the ledges, and the curved graphene lattice of the nanotubes. If this was the case, then one could expect a certain degree of control over the chirality and handedness of the nanotubes. Another question with greater practical implications for future applications of nanotube epitaxy in nanoelectronics concerns the degree of atomic perfection and the electronic properties of the epitaxial nanotubes. Moreover, it is not yet clear whether the atomic steps and rows or nanofacets perturb the electronic properties of the nanotubes. The fact that these epitaxial substrates are bulk dielectric materials has also made their integration and electrical characterization difficult. A possible solution would be to develop a procedure for transferring the organized nanotubes from the epitaxial templates onto silicon wafers, or onto predefined target structures. Progress in these directions should soon enable the realization of many applications. 


\section{Acknowledgements}

First and foremost, I would like to thank all the students and collaborators, whose work is reviewed here: Ariel Ismach, Noam Geblinger, Lior Segev, David Kantorovich, Hyungbin Son, Mildred M. Dresselhaus, Gene Dresselhaus, L. Gustavo Cançado, Ado Jorio, and Lukas Novotny. This research was supported by the Israel Science Foundation, the US-Israel Binational Science Foundation, the Helen and Martin Kimmel Center for Nanoscale Science, and the Legrain, Djanogly, Alhadeff and Perlman foundations.

\section{References}

[1] lijima, S. Helical microtubules of graphitic carbon. Nature 1991, 354, 56-58.

[2] Jorio, A.; Dresselhaus, G.; Dresselhaus, M. S. Carbon nanotubes: Advanced topics in the synthesis, structure, properties and applications, series: Top. Appl. Phys., vol 111. Springer, 2008

[3] Service, R. F. Assembling nanocircuits from the bottom up. Science 2001, 293, 782-785.

[4] Liu, J.; Casavant, M. J.; Cox, M.; Walters, D. A.; Boul, P.; Lu, W.; Rimberg, A. J.; Smith, K. A.; Colbert, D. T.; Smalley, R. E. Controlled deposition of individual singlewalled carbon nanotubes on chemically functionalized templates. Chem. Phys. Lett. 1999, 303, 125-129.

[5] Diehl, M. R.; Yaliraki, S. N.; Beckman, R. A.; Barahona, M.; Heath, J. R. Self-assembled, deterministic carbon nanotube wiring networks. Angew. Chem. Int. Ed. 2002, 41, 353-356.

[6] Krupke, R.; Hennrich, F.; von Lohneysen, H.; Kappes, M. $M$. Separation of metallic from semiconducting singlewalled carbon nanotubes. Science 2003, 301, 344-347.

[7] Joselevich, E.; Lieber, C. M. Vectorial growth of metallic and semiconducting single-wall carbon nanotubes. Nano Lett. 2002, 2, 1137-1141.

[8] Huang, S. M.; Cai, X. Y.; Liu, J. Growth of millimeter-long and horizontally aligned single-walled carbon nanotubes on flat substrates. J. Am. Chem. Soc. 2003, 125, 56365637.

[9] Joselevich, E.; Dai, H. J.; Liu, J.; Hata, K.; Windle, A. H. Carbon nanotube synthesis and organization. Top. Appl. Phys. 2008, 111, 101-164.
[10] Hooks, D. E.; Fritz, T.; Ward, M. D. Epitaxy and molecular organization on solid substrates. Adv. Mater. 2001, 13, 227-241.

[11] Ismach, A.; Kantorovich, D.; Joselevich, E. Carbon nanotube graphoepitaxy: Highly oriented growth by faceted nanosteps. J. Am. Chem. Soc. 2005, 127, 11554 $-11555$.

[12] Smalley, R. E.; Li, Y. B.; Moore, V. C.; Price, B. K.; Colorado, R.; Schmidt, H. K.; Hauge, R. H.; Barron, A. R.; Tour, J. M. Single wall carbon nanotube amplification: En route to a type-specific growth mechanism. J. Am. Chem. Soc. 2006, 128, 15824-15829.

[13] Wang, Y. H.; Kim, M. J.; Shan, H. W.; Kittrell, C.; Fan, H.; Ericson, L. M.; Hwang, W. F.; Arepalli, S.; Hauge, R. H.; Smalley, R. E. Continued growth of single-walled carbon nanotubes. Nano Lett. 2005, 5, 997-1002.

[14] Kim, M. J.; Haroz, E.; Wang, Y.; Shan, H.; Nicholas, N.; Kittrell, C.; Moore, V. C.; Jung, Y.; Luzzi, D.; Wheeler R.; BensonTolle, T.; Fan, H.; Da, S.; Hwang, W. F.; Wainerdi, T. J.; Schmidt, H.; Hauge, R. H.; Smalley, R. E. Nanoscopically flat open-ended single-walled carbon nanotube substrates for continued growth. Nano Lett. 2007, 7, 15-21.

[15] Liu, J.; Rinzler, A. G.; Dai, H. J.; Hafner, J. H.; Bradley, R. K.; Boul, P. J.; Lu, A.; Iverson, T.; Shelimov, K.; Huffman, C. B.; Rodriguez-Macias, F.; Shon, Y. S.; Lee, T. R.; Colbert, D. T.; Smalley, R. E. Fullerene pipes. Science 1998, 280, 12531256.

[16] Su, M.; Li, Y.; Maynor, B.; Buldum, A.; Lu, J. P.; Liu, J. Lattice-oriented growth of single-walled carbon nanotubes. J. Phys. Chem. B 2000, 104, 6505-6508.

[17] Tominaga, M.; Ohira, A.; Kubo, A.; Taniguchi, I.; Kunitake, M. Growth of carbon nanotubes on a gold (111) surface using two-dimensional iron oxide nanoparticle catalysts derived from iron storage protein. Chem. Commun. 2004, 1518-1519.

[18] Derycke, V.; Martel, R.; Radosvljevic, M.; Ross, F. M. R.; Avouris, P. Catalyst-free growth of ordered single-walled carbon nanotube networks. Nano Lett. 2002, 2, 1043 1046.

[19] Ruppalt, L. B.; Albrecht, P. M.; Lyding, J. W. Atomic resolution scanning tunneling microscope study of single-wailed carbon nanotubes on $\operatorname{GaAs}(110)$. J. Vac. Sci. Technol. B 2004, 22, 2005-2007.

[20] Ruppalt, L. B.; Albrecht, P. M.; Lyding, J. W. UHV-STM study of single-walled carbon nanotubes applied to the 
GaAs(110) and InAs(110) surfaces. J. Phys. IV 2006, 132, 31-34.

[21] Ismach, A.; Segev, L.; Wachtel, E.; Joselevich, E. Atomicstep-templated formation of single wall carbon nanotube patterns. Angew. Chem. Int. Ed. 2004, 43, 6140-6143.

[22] Han, S.; Liu, X. L.; Zhou, C. W. Template-free directional growth of single-walled carbon nanotubes on a- and r-plane sapphire. J. Am. Chem. Soc. 2005, 127, 52945295.

[23] Ago, H.; Nakamura, K.; Ikeda, K.; Uehara, N.; Ishigami, N.; Tsuji, M. Aligned growth of isolated single-walled carbon nanotubes programmed by atomic arrangement of substrate surface. Chem. Phys. Lett. 2005, 408, 433438.

[24] Ago, H.; Uehara, N.; Ikeda, K.; Ohdo, R.; Nakamura, K.; Tsuji, M. Synthesis of horizontally-aligned single-walled carbon nanotubes with controllable density on sapphire surface and polarized Raman spectroscopy. Chem. Phys. Lett. 2006, 421, 399-403.

[25] Yu, Q. K.; Qin, G. T.; Li, H.; Xia, Z. H.; Nian, Y. B.; Pei, S. S. Mechanism of horizontally aligned growth of single-wall carbon nanotubes on r-plane sapphire. J. Phys. Chem. $B$ 2006, 110, 22676-22680.

[26] Liu, X. L.; Han, S.; Zhou, C. W. Novel nanotube-oninsulator (NOI) approach toward single-walled carbon nanotube devices. Nano Lett. 2006, 6, 34-39.

[27] Maret, M.; Hostache, K.; Schouler, M. C.; Marcus, B.; Roussel-Dherbey, F.; Albrecht, M.; Gadelle, P. Oriented growth of single-walled carbon nanotubes on a MgO(001) surface. Carbon 2007, 45, 180-187.

[28] Souza, M.; Jorio, A.; Fantini, C.; Neves, B. R. A.; Pimenta, M. A.; Saito, R.; Ismach, A.; Joselevich, E.; Brar, V. W.; Samsonidze, G. G.; Dresselhaus, G.; Dresselhaus, M. S. Single- and double-resonance Raman G-band processes in carbon nanotubes. Phys. Rev. B 2004, 69, 241403.

[29] Ismach, A.; Kantorovich, D.; Berson, J.; Geblinger, N.; Segev, L.; Wachtel, E.; Son, H.; Jorio, A.; Dresselhaus, M. S.; Dresselhaus, G.; Joselevich, E. Epitaxial modes of carbon nanotube growth on vicinal $\alpha-\mathrm{Al}_{2} \mathrm{O}_{3}(0001)$ surfaces. Unpublished.

[30] Kocabas, C.; Hur, S. H.; Gaur, A.; Meitl, M. A.; Shim, M.; Rogers, J. A. Guided growth of large-scale, horizontally aligned arrays of single-walled carbon nanotubes and their use in thin-film transistors. Small 2005, 1, 11101116

[31] Kocabas, C.; Shim, M.; Rogers, J. A. Spatially selective guided growth of high-coverage arrays and random networks of single-walled carbon nanotubes and their integration into electronic devices. J. Am. Chem. Soc. 2006, 128, 4540-4541.

[32] Kocabas, C.; Kang, S. J.; Ozel, T.; Shim, M.; Rogers, J. A. Improved synthesis of aligned arrays of single-walled carbon nanotubes and their implementation in thin film type transistors. J. Phys. Chem. C 2007, 111, 1787917886.

[33] Yuan, D.; Ding, L.; Chu, H. B.; Feng, Y. Y.; McNicholas, T. P.; Liu, J. Horizontally aligned single-walled carbon nanotube on quartz from a large variety of metal catalysts. Nano Lett. 2008, 8, 2576-2579.

[34] Ding, L.; Tselev, A.; Wang, J. Y.; Yuan, D. N.; Chu, H. B.; McNicholas, T. P.; Li, Y.; Liu, J. Selective growth of wellaligned semiconducting single-wall carbon nanotubes. Nano Lett. 2009, 9, 800-805.

[35] Smith, H. I.; Flanders, D. C. Oriented crystal-growth on amorphous substrates using artificial surface-relief gratings. Appl. Phys. Lett. 1978, 32, 349-350.

[36] Segalman, R. A.; Yokoyama, H.; Kramer, E. J. Graphoepitaxy of spherical domain block copolymer films. Adv. Mater. 2001, 13, 1152-1155.

[37] Rueckes, T.; Kim, K.; Joselevich, E.; Tseng, G. Y.; Cheung, C. L.; Lieber, C. M. Carbon nanotube-based nonvolatile random access memory for molecular computing. Science 2000, 289, 94-97.

[38] Refael, G.; Heo, J. S; Bockrath, M. Sagnac interference in carbon nanotube loops. Phys. Rev. Lett. 2007, 98, 246803.

[39] Ismach, A.; Joselevich, E. Orthogonal self-assembly of carbon nanotube crossbar architectures by simultaneous graphoepitaxy and field-directed growth. Nano Lett. 2006, 6, 1706-1710.

[40] Geblinger, N. MSc Thesis, Weizmann Institute of Science, Israel, 2008.

[41] Gebliner, N.; Ismach, A.; Joselevich, E. Self-organized nanotube serpentines. Nat. Nanotechnol. 2008, 3, 195200.

[42] Wang, Y. H.; Maspoch, D.; Zou, S. L.; Schatz, G. C.; Smalley, R. E.; Mirkin, C. A. Controlling the shape, orientation, and linkage of carbon nanotube features with nano affinity templates. Proc. Natl. Acad. Sci. U.S.A. 2006, 103, 2026-2031.

[43] Tsukruk, V. V.; Ko, H.; Peleshanko, S. Nanotube surface arrays: Weaving, bending, and assembling on patterned

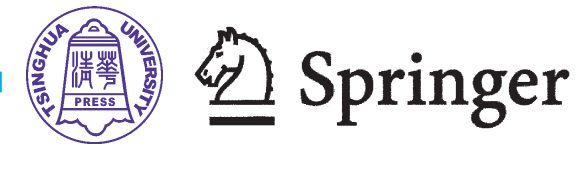


silicon. Phys. Rev. Lett. 2004, 92, 065502.

[44] Duggal, R.; Pasquali, M. Dynamics of individual singlewalled carbon nanotubes in water by real-time visualization. Phys. Rev. Lett. 2006, 96, 246104.

[45] Hertel, T.; Walkup, R. E.; Avouris, P. Deformation of carbon nanotubes by surface van der Waals forces. Phys. Rev. B 1998, 58, 13870-13873.

[46] Whitesides, G. M.; Grzybowski, B. Self-assembly at all scales. Science 2002, 295, 2418-2421.

[47] Rabani, E.; Reichman, D. R.; Geissler, P. L.; Brus, L. E. Drying-mediated self-assembly of nanoparticles. Nature 2003, 426, 271-274.

[48] Nicolis, G.; Prigogine, I. Self-Organization in NonEquilibrium Systems: From Dissipative Structures to Order Through Fluctuations; John Wiley: New York, 1977.

[49] Kurin-Csorgei, K.; Epstein, I. R.; Orban, M. Systematic design of chemical oscillators using complexation and precipitation equilibria. Nature 2005, 433, 139-142.

[50] Bachtold, A.; Fuhrer, M. S.; Plyasunov, S.; Forero, M.; Anderson, E. H.; Zettl, A.; McEuen, P. L.; Scanned probe microscopy of electronic transport in carbon nanotubes. Phys. Rev. Lett. 2000, 84, 6082-6085.

[51] Javey, A.; Guo, J.; Wang, Q.; Lundstrom, M.; Dai, H. J. Ballistic carbon nanotube field-effect transistors. Nature
2003, 424, 654-657.

[52] Kang, S. J.; Kocabas, C.; Ozel, T.; Shim, M.; Pimparkar, N.; Alam, M. A.; Rotkin, S. V.; Rogers, J. A. Highperformance electronics using dense, perfectly aligned arrays of single-walled carbon nanotubes. Nat. Nanotechnol. 2007, 2, 230-236.

[53] Wang, Y.; Kempa, K.; Kimball, B.; Carlson, J. B.; Benham, G.; Li, W. Z.; Kempa, T.; Rybczynski, J.; Herczynski, A.; Ren, Z. F. Receiving and transmitting light-like radio waves: Antenna effect in arrays of aligned carbon nanotubes. Appl. Phys. Lett. 2004, 85, 2607-2609.

[54] Springel, V.; Frenk, C. S.; White, S. D. M. The large-scale structure of the universe. Nature 2006, 440, 1137-1144.

[55] Werner, B. T.; Complexity in natural landform patterns. Science 1999, 284, 102-104.

[56] Vollbrecht, E.; Springer, P. S.; Goh, L.; Buckler, E. S.; Martienssen, R. Architecture of floral branch systems in maize and related grasses. Nature 2005, 436, 1119 1126.

[57] Lumelsky, N.; Blondel, O.; Laeng, P.; Velasco, I.; Ravin, R.; McKay, R. Differentiation of embryonic stem cells to insulin-secreting structures similar to pancreatic islets. Science 2001, 292, 1389-1394. 